\title{
Prognostic evaluation of child patients with infectious encephalitis through AEEG and REEG
}

\author{
YUJUN LIN ${ }^{1}$, GE ZHANG $^{2}$, YAN WANG $^{3}$, JIANJUN CHAI $^{4}$, XIUFANG JIANG $^{2}, \mathrm{CONG} \mathrm{LI}^{3}$ and HUI XU ${ }^{5}$ \\ ${ }^{1}$ Department of Electrophysiology, Yantaishan Hospital of Yantai, Yantai, Shandong 264000; \\ Departments of ${ }^{2}$ Obstetrics, ${ }^{3}$ Public Health and ${ }^{4}$ Neurosurgery, People's Hospital of Zhangqiu, Jinan, Shandong 250200; \\ ${ }^{5}$ Department of Internal Medicine, Weifang People's Hospital, Weifang, Shandong 261000, P.R. China
}

Received April 2, 2018; Accepted October 2, 2018

DOI: $10.3892 /$ etm.2018.6882

\begin{abstract}
This study investigated prognostic evaluation of child patients with viral encephalitis through ambulatory electroencephalogram (AEEG) and regular electroencephalogram (REEG). A total of 94 child patients who were clinically diagnosed with viral encephalitis in Yantaishan Hospital of Yantai from May 2010 to July 2014, was examined with AEEG and REEG, respectively and randomly divided into AEEG group $(n=47)$ and REEG group $(n=47)$. The probabilities of detecting abnormal electroencephalographic activities with two examination methods were compared. The detection rates of abnormal electroencephalographic activities with AEEG and REEG were 80.0 and $65.0 \%$, respectively, with significant differences $(\mathrm{P}<0.05)$; the probabilities of detecting epileptiform discharge with AEEG and REEG were 42.5 and 6.3\%, respectively, with significant differences $(\mathrm{P}<0.05)$. The hospitalization time and time of electroencephalogram (EEG) restoring to normal of child patients with encephalitis in the REEG group were significantly longer than those of child patients with encephalitis in the AEEG group, with statistically significant differences $(\mathrm{P}<0.05)$. Among child patients in the AEEG group, the incidence rate of severe illness was $2.1 \%$, and both the incidence rates of clinical recurrence and of sequela were 0 . Among child patients in the REEG group, the incidence rate, clinical recurrence rate and incidence rate of sequela were $8.5,12.7$ and $8.5 \%$, respectively, with statistically significant differences $(\mathrm{P}<0.05)$. To some extent, the EEG abnormality reflects the disorder degree of brain environment of child patients with viral encephalitis. The treatment effect and prognosis of child patients with viral encephalitis can be clinically evaluated based on EEG monitoring results of child patients, which has a certain clinical guiding significance.
\end{abstract}

Correspondence to: Dr Hui Xu, Department of Internal Medicine, Weifang People's Hospital, 151 Guangwen Street, Weifang, Shandong 261000, P.R. China

E-mail: hr26us@163.com

Key words: AEEG, REEG, viral encephalitis, prognosis, epileptiform discharge
AEEG has important significance to the auxiliary diagnosis of viral encephalitis, with higher sensitivity than REEG.

\section{Introduction}

Currently, infectious encephalitis belongs to virosis of the central nervous system and is common in child patients (1). Bacterium, virus, fungus and mycoplasma can lead to viral encephalitis with inflammatory lesions, and the most prevalent encephalitis mainly consists of viral encephalitis. The child patients' main symptoms include headache, fever and vomiting, and child patients with severe viral encephalitis suffer from stress injury (2-4). Stress response is a kind of non-specific systemic reaction occurring in organisms, and mild stress response can improve the adaptability of human body, while excessive stress response exacerbates function impairment of the human body (5).

Clinical treatment methods for viral encephalitis have obtained unfavourable effects and mainly aim at clinical symptoms of child patients. When it is clinically found that the brain environment disorder of child patients with viral encephalitis leads to the corresponding changes in electroencephalograms (EEGs), changes in disease conditions of such child patients can be analyzed based on changes in EEG. However, response results of different EEGs vary, and ambulatory electroencephalogram (AEEG) has higher specificity and diagnostic value than regular electroencephalogram (REEG) (6-8). Therefore, this study aims to evaluate the prognosis of child patients with viral encephalitis through AEEG and REEG.

\section{Patients and methods}

Clinical information. Child patients who were primarily diagnosed with viral encephalitis 3 days after being admitted to Yantaishan Hospital of Yantai (Yantai, China) from May 2010 to July 2014 were selected to receive $24 \mathrm{~h}$ computed tomography (CT), magnetic resonance imaging (MRI) and cerebrospinal fluid (CSF) examinations, and the diagnosis criteria for viral encephalitis were proposed with reference to literature (5): i) patients with clinical signs of brain parenchyma damage due to virus; ii) patients with inflammatory changes in CSF; iii) patients without space-occupying lesion signs in brain CT and MRI; iv) patients with nervous system 
disease, multiple sclerosis neurological disease, and neurologic demyelinating disease were excluded. There were 94 child patients with viral encephalitis, in total. Of them, there were 53 males and 41 females aged 4-11 years and an average age of 7.40 \pm 1.71 years; the disease course lasted 1-3 days with an average of $1.85 \pm 0.80$ days. They were randomly divided into the REEG group $(n=47)$ and AEEG group $(n=47)$ by contrast, and the two groups did not have statistical differences in the information on age and sex of patients. Families and guardians of all child patients signed the informed consent. The study was approved by the Ethics Committee of Yantaishan Hospital of Yantai.

EEG monitoring. REEG group adopted a 16-lead conventional electroencephalograph (Dantec Dynamics, Skovlunde, Denmark), and the electrodes were placed on scalp strictly in accordance with the international $10-20 \%$ system to conduct regular monopolar and bipolar lead tracing as per $30 \mathrm{~min} / \mathrm{time}$. Moreover, the experiment was induced by coordinating routine eye-closing reaction and overventilation of patients. The AEEG group used an 8-lead ambulatory electroencephalograph (NCC, Shanghai, China), and the electrodes were placed on scalp strictly in accordance with the international $10-20 \%$ system to conduct regular monopolar lead tracing for $24 \mathrm{~h}$ real-time monitoring.

Observation criteria and assessment indicators. The abnormity rates of different types of EEGs during hospitalization were identified. First of all, EEG abnormities were analyzed with reference to Clinical Electroencephalography (9). The abnormity degree in this experiment was divided into three levels: mild, moderate and severe. Mildly abnormal EEG mainly showed $\alpha$ wave with middle-low amplitude $\theta$ wave and extremely few $\delta$ waves; moderately abnormal EEG mainly presented middle-low amplitude $\theta$ wave with active $\delta$ wave; severely abnormal EEG exhibited high-amplitude $\theta$ wave and $\delta$ wave rhythmic activity. At last, the hospitalization time, time of EEG restoring to normal and incidence rates of clinical reoccurrence and sequela were calculated based on results of different types of electroencephalograph. The prognoses of child patients with normal and abnormal EEGs in the two groups were analyzed. Furthermore, the changes in EEG of child patients were analyzed to evaluate prognoses of child patients and clinical values.

Statistical analysis. The data obtained in this study were statistically analyzed with Statistical Product and Service Solutions (SPSS) 19.0 software package (SPSS Inc., Chicago, IL, USA), the measurement data were expressed as mean \pm standard error. The inter-group data were tested using the Kolmogorov-Smirnov (K-S) method, and the enumeration data were verified by Chi-square test. $\mathrm{P}<0.05$ was considered to indicate a statistically significant difference.

\section{Results}

Comparison of abnormity rate between AEEG and REEG. In the REEG group, 25 cases of EEG abnormity were found with the abnormity rate of $53.2 \%$, while in the AEEG group, 38 cases of EEG abnormity were found with the abnormity
Table I. Comparison of abnormity rate between the AEEG and REEG groups.

\begin{tabular}{|c|c|c|c|c|c|c|}
\hline \multirow[b]{2}{*}{$\begin{array}{l}\text { EEG } \\
\text { abnormity }\end{array}$} & \multicolumn{2}{|c|}{ AEEG (n=47) } & \multicolumn{2}{|c|}{ REEG (n=47) } & \multirow[b]{2}{*}{$\chi^{2}$} & \multirow[b]{2}{*}{ P-value } \\
\hline & $\mathrm{n}$ & $\begin{array}{c}\text { Abnormity } \\
\text { rate }(\%)\end{array}$ & $\mathrm{n}$ & $\begin{array}{c}\text { Abnormity } \\
\text { rate }(\%)\end{array}$ & & \\
\hline Mild & 7 & 14.9 & 6 & 12.8 & 0.089 & 0.765 \\
\hline Moderate & 11 & 23.4 & 14 & 29.8 & 0.490 & 0.484 \\
\hline Severe & 20 & 42.6 & 5 & 10.6 & 9.889 & 0.002 \\
\hline Total & 38 & 80.9 & 25 & 53.2 & 6.931 & 0.004 \\
\hline
\end{tabular}

AEEG, ambulatory electroencephalogram; REEG, regular electroencephalogram; EEG, electroencephalogram.

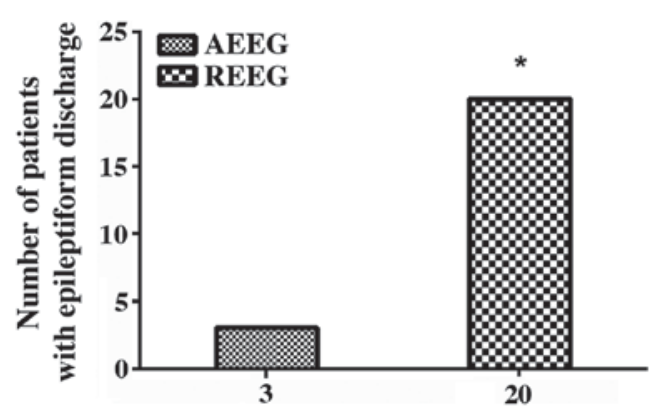

Figure 1. Comparison of epileptiform discharge between the AEEG and REEG groups. The detection rate detected by AEEG was higher than that detected by REEG; *P<0.05. AEEG, ambulatory electroencephalogram; REEG, regular electroencephalogram.

rate of $80.9 \%$. The comparison of abnormity rate between child patients in the REEG and AEEG groups was statistically significant $(\mathrm{P}<0.05)$. The comparison of abnormity rate between severe child patients in the two groups was statistically significant $(\mathrm{P}<0.05)$. The comparison of abnormity rates between mild and moderate child patients in the two groups did not indicate statistical significance $(\mathrm{P}>0.05)$ (Table I).

Comparison of epileptiform discharge between the AEEG and REEG groups. In the REEG group, only 3 child patients with epileptiform discharge were monitored with the detection rate of $6.4 \%$. In the AEEG group, 20 cases of such child patients were monitored with the detection rate of $42.6 \%$. According to statistical analysis, the two methods had a significant difference $(\mathrm{P}<0.05)($ Fig. 1).

Comparison of clinical indicators of child patient EEG in the two groups. As the abnormal fluctuation of child patient EEGs became more significant, the hospitalization time and time of EEG restoring to normal were increased correspondingly. In the AEEG group, they were substantially shortened, and statistically different from those in the REEG group $(\mathrm{P}<0.001)$ (Table II).

Comparison of prognoses among child patients. According to the study, in the AEEG group, the incidence rate of severe disease among child patients was $2.1 \%$, and both incidence 
Table II. Comparison of clinical indicators of child patient EEG in the two groups.

\begin{tabular}{|c|c|c|c|c|c|c|c|}
\hline Groups & $\begin{array}{c}\text { Abnormity degree } \\
\text { of EEG }\end{array}$ & $\begin{array}{l}\text { AEEG } \\
\text { group } \\
\text { (d) }\end{array}$ & F-value & P-value & $\begin{array}{l}\text { REEG } \\
\text { group } \\
\text { (d) }\end{array}$ & F-value & P-value \\
\hline Hospitalization time & $\begin{array}{c}\text { Mild } \\
\text { Moderate } \\
\text { Severe }\end{array}$ & $\begin{array}{r}7.44 \pm 3.84 \\
12.61 \pm 4.58 \\
19.17 \pm 4.97\end{array}$ & 12.21 & $<0.001$ & $\begin{array}{r}9.18 \pm 3.51 \\
17.61 \pm 5.14 \\
26.51 \pm 4.21\end{array}$ & 32.65 & $<0.001$ \\
\hline $\begin{array}{l}\text { Time of EEG restoring } \\
\text { to normal }\end{array}$ & $\begin{array}{c}\text { Mild } \\
\text { Moderate } \\
\text { Severe }\end{array}$ & $\begin{array}{l}13.45 \pm 3.74 \\
18.57 \pm 4.28 \\
24.16 \pm 5.27\end{array}$ & 17.39 & $<0.001$ & $\begin{array}{l}18.37 \pm 4.67 \\
23.49 \pm 4.87 \\
28.67 \pm 5.62\end{array}$ & 4.35 & 0.026 \\
\hline
\end{tabular}

EEG, electroencephalogram; AEEG, ambulatory electroencephalogram; REEG, regular electroencephalogram.

Table III. Comparison of prognoses among child patients $(\mathrm{n}, \%)$.

\begin{tabular}{lccccc}
\hline Groups & $\mathrm{n}$ & AEEG & REEG & $\chi^{2}$ & P-value \\
\hline $\begin{array}{l}\text { Incidence rate of } \\
\text { severe disease }\end{array}$ & 5 & $1(2.1 \%)$ & $4(8.5 \%)$ & 0.845 & 0.361 \\
$\begin{array}{l}\text { Incidence rate of } \\
\text { clinical reoccurrence }\end{array}$ & 6 & 0 & $(12.7 \%)$ & 4.451 & 0.026 \\
$\begin{array}{l}\text { Incidence rate of } \\
\text { sequela }\end{array}$ & 4 & 0 & $(8.5 \%)$ & 2.350 & 0.117 \\
\hline
\end{tabular}

AEEG, ambulatory electroencephalogram; REEG, regular electroencephalogram.

rates of clinical reoccurrence and sequela were 0 . By contrast, in the REEG group, these rates were 8.5, 12.7 and $8.5 \%$, respectively. Through comparisons, the two groups had a statistical difference in the incidence rate of clinical reoccurrence $(\mathrm{P}<0.05)$, rather than in the incidence rates of severe disease and sequela $(\mathrm{P}>0.05)$ (Table III).

\section{Discussion}

Encephalitis is a nervous functional disorder, and inflammation tends to occur in immunological diseases, intoxication, metabolic encephalopathy, cancer, vascular diseases or viral diseases (10-12). Infectious encephalitis mainly consists of three types: viral encephalitis, suppurative meningitis and mycoplasma encephalitis (13). Viral encephalitis belongs to a kind of inflammation due to the invasion of virus into brain parenchyma. Patients are infected with virus and thus, brain parenchyma has virus-induced lesions. As a result, patients present certain clinical symptoms, such as headache, nausea and fever $(14,15)$.

EEG detecting, a simple method to monitor brain functions, can relatively correctly monitor abnormal electroencephalographic activities and brain damage and help clinical doctors take the corresponding timely treatment measures for child patients (16). REEG and AEEG are the most common clinical instruments, currently and often used for the auxiliary diagnosis of viral encephalitis $(17,18)$. REEG has a significant effect on the diagnosis of viral encephalitis and can timely reflect the moderate and severe abnormities of child patients, which has an important clinical significance to viral encephalitis. However, AEEG can more obviously and accurately detect abnormal discharge due to brain damage (19). The main reason for the difficulty in detecting abnormal discharge of patients with viral encephalitis with REEG is that the monitoring time is short. On the contrary, AEEG can monitor the changes in brain wave for $24 \mathrm{~h}$, and it is easier to detect the abnormal discharge, effectively avoiding clinical misdiagnoses and diagnostic errors (20).

It was found in this study that REEG detected 22 normal child patients and 25 abnormal child patients with the abnormity rate of $53.2 \%$. AEEG detected 9 normal child patients and 38 abnormal child patients with the abnormity rate of $80.9 \%$, indicating the abnormity rate in the AEEG group is significantly higher than that in the REEG group $(\mathrm{P}<0.05)$. Child patients with viral encephalitis have continuous and paroxysmal abnormal brain waves, and abnormal electroencephalographic activities can be traced better by AEEG for the long monitoring time and continuous monitoring (21). The REEG group had a higher detection rate of moderately abnormal child patients, but lower detection rates of mildly and severely abnormal child patients. In the AEEG group, the detection rate of severely abnormal child patients were significantly higher than that of mildly abnormal child patients. Through comparison, the detection rate of severely abnormal child patients in the AEEG group was obviously higher than that in the REEG group $(\mathrm{P}<0.05)$. This fully indicates that two different EEGs have varying detection rates for encephalitis in different courses. In this study, REEG cannot more favourably discriminate between mild and moderate child patients with viral encephalitis and between mild and severe child patients. In contrast, AEEG can relatively successfully discriminate severe child patients with viral encephalitis from moderate and mild ones with a high detection rate. In the REEG group, 3 child patients with epileptiform discharge were detected with the detection rate of $6.4 \%$, while in the AEEG group, 20 cases of epileptiform discharge were detected with the detection rate of $42.6 \%(\mathrm{P}<0.05)$. Generally, in the position application of epileptiform discharge, monopolar lead equipment has a better positioning effect than bipolar one and relatively low 
amplitude and distortion degree. The hospitalization time of child patients and time of EEG restoring to be normal were calculated based on the study results. The statistical results showed that with the abnormity degree of EEG deepened, the hospitalization time of child patients and time of EEG restoring to normal were correspondingly prolonged. Both the REEG and AEEG groups had differences in the time of restoring and hospitalization time of child patients $(\mathrm{P}<0.05)$. At last, the prognoses of child patients were analyzed, and it was found that the comparison of the incidence rate of clinical reoccurrence between the two groups was statistically significant $(\mathrm{P}<0.05)$. The comparison of incidence rates of severe disease and sequela between the two groups were not statistically significant $(\mathrm{P}>0.05)$. The abnormity of EEG reflects the damage degree of child patient brain parenchyma, and the more abnormal the amplitude of fluctuation is, the more severe the damage becomes and the larger the opportunity of sequela is. As the disease conditions of child patients were improved, the clinical symptoms were correspondingly decreased, and the abnormity rate of EEG was significantly decreased. Therefore, EEG restoring to normal can be taken as one of child patient disease condition improvement indicators (22). However, the improvement of child patients cannot be confirmed only based on EEG restoring to normal, and multiple clinical indicators should be considered to assess disease conditions of child patients.

There are also certain defects in this study. For example, whether the medication of child patients before visiting doctors has an effect on EEG should be confirmed, and a larger number of samples need analyzing to prove this. Future studies will be conducted to further verify the conclusion of this study.

In conclusion, EEG can be used for the auxiliary diagnosis of child patients with viral encephalitis, and AEEG is superior to REEG, which has higher sensitivity and can be taken as an indicator for the auxiliary diagnosis of viral encephalitis and the prognosis of child patients.

\section{Acknowledgements}

Not applicable.

\section{Funding}

No funding was received.

\section{Availability of data and materials}

The datasets used and/or analyzed during the present study are available from the corresponding author on reasonable request.

\section{Authors' contributions}

YL and CW conceived and designed the study. GZ, CL, JC and XJ were responsible for the collection and analysis of the patient data. YL and YW helped with EEG monitoring. YL, GZ and CL interpreted the data and drafted the manuscript. JC and CW revised the manuscript critically for important intellectual content. All authors read and approved the final manuscript.

\section{Ethics approval and consent to participate}

The study was approved by the Ethics Committee of Yantaishan Hospital of Yantai (Yantai, China). Signed informed consents were obtained from the parents or guardians of the child patients.

\section{Patient consent for publication}

Not applicable.

\section{Competing interests}

The authors declare that they have no competing interests.

\section{References}

1. Armangue T, Leypoldt F and Dalmau J: Autoimmune encephalitis as differential diagnosis of infectious encephalitis. Curr Opin Neurol 27: 361-368, 2014.

2. Adenot M, Frobert E, Blanchard G, Morel B, Perrot L, Floret D and Javouhey E: Clinical presentation of severe viral encephalitis with known causative agents in children: A retrospective study on 16 patients hospitalized in a pediatric intensive care unit (2008-2011). J Child Neurol 29: 1508-1518, 2014.

3. Daxboeck F, Blacky A, Seidl R, Krause R and Assadian O: Diagnosis, treatment, and prognosis of Mycoplasma pneumoniae childhood encephalitis: Systematic review of 58 cases. J Child Neurol 19: 865-871, 2004.

4. Pelkonen T, Sarajuuri A, Rautanen T, Sinkkonen S and Jero J: Meningoencephalitis and otitis media in a child with Mycoplasma pneumoniae infection. Acta Otolaryngol Case Rep 2: 1-4, 2017.

5. Taba P, Schmutzhard E, Forsberg P, Lutsar I, Ljøstad U, Mygland Å, Levchenko I, Strle F and Steiner I: EAN consensus review on prevention, diagnosis and management of tick-borne encephalitis. Eur J Neurol 24: 1214-e61, 2017.

6. Domingues RB: Treatment of viral encephalitis. Cent Nerv Syst Agents Med Chem 9: 56-62, 2009.

7. Spencer DC, Sun FT, Brown SN, Jobst BC, Fountain NB, Wong VS, Mirro EA and Quigg M: Circadian and ultradian patterns of epileptiform discharges differ by seizure-onset location during long-term ambulatory intracranial monitoring. Epilepsia 57: 1495-1502, 2016.

8. Seneviratne U, Mohamed A, Cook M and D'Souza W: The utility of ambulatory electroencephalography in routine clinical practice: A critical review. Epilepsy Res 105: 1-12, 2013.

9. Kiloh LG, McComas AJ and Osselton JW: Clinical Electroencephalography. 3rd edition. Butterworth-Heinemann, Oxford, UK, 1972.

10. Venkatesan A, Tunkel AR, Bloch KC, Lauring AS, Sejvar J, Bitnun A, Stahl JP, Mailles A, Drebot M, Rupprecht CE, et al; International Encephalitis Consortium: Case definitions, diagnostic algorithms, and priorities in encephalitis: Consensus statement of the international encephalitis consortium. Clin Infect Dis 57: 1114-1128, 2013.

11. Armangue T, Leypoldt F and Dalmau J: Autoimmune encephalitis as differential diagnosis of infectious encephalitis. Curr Opin Neurol 27: 361-368, 2014.

12. Pillai SC, Hacohen Y, Tantsis E, Prelog K, Merheb V, Kesson A, Barnes E, Gill D, Webster R, Menezes M, et al: Infectious and autoantibody-associated encephalitis: Clinical features and long-term outcome. Pediatrics 135: e974-e984, 2015.

13. Graus F, Titulaer MJ, Balu R, Benseler S, Bien CG, Cellucci T, Cortese I, Dale RC, Gelfand JM, Geschwind M, et al: A clinical approach to diagnosis of autoimmune encephalitis. Lancet Neurol 15: 391-404, 2016.

14. Qian Y, Wong CC, Lai SC, Lin ZH, Zheng WL, Zhao H, Pan KH, Chen SJ and Si JM: Klebsiella pneumoniae invasive liver abscess syndrome with purulent meningitis and septic shock: A case from mainland China. World J Gastroenterol 22: 2861-2866, 2016. 
15. Kennedy PGE, Quan PL and Lipkin WI: Viral encephalitis of unknown cause: Current perspective and recent advances. Viruses 9: 138, 2017.

16. Mohammad SS, Soe SM, Pillai SC, Nosadini M, Barnes EH, Gill D and Dale RC: Etiological associations and outcome predictors of acute electroencephalography in childhood encephalitis. Clin Neurophysiol 127: 3217-3224, 2016.

17. Namekawa K, Mori H, Tabata H, Miyahara H, Minote M, Nishida A and Shindo K: Extreme delta brush in electroencephalogram may be nonspecific to the anti-NMDAR encephalitis. Neurology 88: 223, 2017.

18. Fangsaad T, Assawabumrungkul S and Visudtibhan A: Clinical course and long-term outcome in children with alteration of consciousness underwent continuous EEG monitoring: A prospective observational study in Thailand. J Clin Neurosci 47: 93-96, 2018.

19. Qi XK, Liu JG, Li CQ and Ning BO: 27 cases of atypical viral encephalitis with obvious psychiatric symptom but negative auxiliary diagnosis. J Neurol Sci 357: e115-e116, 2015.
20. Moeller JJ, Tu B and Bazil CW: Quantitative and qualitative analysis of ambulatory electroencephalography during mild traumatic brain injury. Arch Neurol 68: 1595-1598, 2011

21. Lawley A, Evans S, Manfredonia F and Cavanna AE: The role of outpatient ambulatory electroencephalography in the diagnosis and management of adults with epilepsy or nonepileptic attack disorder: A systematic literature review. Epilepsy Behav 53: 26-30, 2015.

22. Gold JJ, Crawford JR, Glaser C, Sheriff H, Wang S and Nespeca M: The role of continuous electroencephalography in childhood encephalitis. Pediatr Neurol 50: 318-323, 2014.

(i) (9) This work is licensed under a Creative Commons Attribution-NonCommercial-NoDerivatives 4.0 International (CC BY-NC-ND 4.0) License. 